\title{
Conhecimentos produzidos acerca do crack: uma incursão nas dissertações e teses brasileiras
}

\author{
Knowledge derived from studies on crack: \\ an incursion into Brazilian dissertations and theses
}

\author{
Diego Schaurich Rodrigues ${ }^{1}$ \\ Dirce Stein Backes ${ }^{2}$ \\ Hilda Maria Barbosa de Freitas ${ }^{2}$ \\ Claudia Zamberlan ${ }^{2}$ \\ Maria Helena Gelhen ${ }^{2}$ \\ Juliana Silveira Colomé ${ }^{2}$
}

${ }^{1}$ Departamento de Enfermagem, Centro de Ciências da Saúde, Universidade Federal do Maranhão. Urbano Santos s/ n, Centro. 65900-410 Imperatriz MA. eu_diegosch@hotmail.com ${ }^{2}$ Conselho de Áreas, Centro Universitário Franciscano
Abstract This is a systematic review based on the integrative review method, which sought to analyze the characteristics of knowledge produced by studies on crack, in Brazilian Master's and Doctoral courses. The investigation comprised 33 studies (18 dissertations and 15 theses). Among them, $51.5 \%$ were from the Health Science area with emphasis on the Postgraduate Program in Psychiatry (and Medical Psychology), which provided five dissertations/theses. Most of the knowledge on the epidemic (51.5\%) are from the Universidade Federal de São Paulo and Universidade de São Paulo, with the largest number of studies $(81.8 \%)$ concentrated in the southeast. The themes most analyzed were: organic alterations, drug trafficking and crack use, HIV/Aids, types and strategies of treatment. The results showed that Brazilian stricto sensu knowledge about crack is still incipient, sketchy and ineffectual, albeit promising due to demands and implications that this epidemic imposes upon society.

Key words Cocaine, Crack, Illegal drugs, Knowledge management for research in health
Resumo Trata-se de um estudo do tipo revisão sistemática, com base na modalidade revisão integrativa, que objetivou analisar as características do conhecimento produzido sobre o crack pelos cursos de mestrado e doutorado brasileiros. O corpus de investigação foi composto por 33 trabalhos (18 dissertações e 15 teses), sendo que 51,5\% dos estudos pertencem à área das Ciências da Saúde, com destaque para o Programa de Pós-Graduação de Psiquiatria (e Psicologia Médica) que produziu cinco dissertações/teses. Os saberes acerca da epidemia são oriundos, em sua maioria (51,5\%), da Universidade Federal de São Paulo e da Universidade de São Paulo, tendo a região Sudeste concentrado $81,8 \%$ dos estudos. As temáticas mais investigadas foram: alterações orgânicas, tráfico de drogas e consumo de crack, HIV/aids, e modelos e estratégias de tratamento. Concluiu-se que o conhecimento "stricto sensu" brasileiro sobre o crack é ainda incipiente, pontual e pouco resolutivo, embora promissor frente às demandas e implicações que esta epidemia impõe à sociedade.

Palavras-chave Cocaina, Crack, Drogas ilícitas, Gestão do conhecimento para a pesquisa em saúde 


\section{Introdução}

Uma das problemáticas que vem chamando a atenção, tanto do poder público quanto dos órgãos de saúde, universidades e sociedade em geral, é o crescimento do consumo de drogas psicotrópicas, especialmente do crack (pedra formada da mistura da pasta básica da cocaína com bicarbonato de sódio) $)^{1}$. Relatos encontrados na literatura $^{1,2}$ indicam que esta droga surgiu, mais precisamente, na década de 80 do século XX, nos Estados Unidos da América. No Brasil, a primeira apreensão ocorreu em 1991, período em que grande parte das atenções governamentais estava voltada para os usuários de drogas injetáveis (devido ao aumento da infecção pelo Vírus da Imunodeficiência Humana - HIV e do adoecimento pela Síndrome da Imunodeficiência Adquirida - AIDS). Estes mesmos relatos apontam que o uso do crack se alastrou em decorrência da tolerância e/ou efeitos agradáveis, fácil administração, custo baixo, alto potencial de dependência, via segura em relação à infecção pelo HIV, dentre outros.

Em virtude dos efeitos estimulantes e prazerosos, aliados aos baixos custos da comercialização ilegal, o crack rapidamente se espalhou pelo território brasileiro, nas mais diferentes classes sociais, podendo ser percebido no cotidiano como um grave problema público que apresenta proporções ainda pouco conhecidas e de grande impacto familiar e social ${ }^{1}$. Tem-se, assim, de um lado o usuário de crack (e sua família) em situação de vulnerabilidade e fragilidade e, de outro, a necessidade de um tratamento complexo com abordagem multiprofissional e interdisciplinar, em serviços ainda pouco estruturados e experientes no atendimento a esta clientela.

Aliar intervenções específicas com vistas a reduzir as manifestações diretas do uso/abuso da droga (farmacoterapia, psicoterapia) com ações mais gerais voltadas a minimizar os prejuízos secundários ao processo de drogadição (estratégias de prevenção à recaída), constituem outros desafios. Os sucessos do tratamento e da reabilitação do usuário de crack exigem, também, políticas educacionais e assistenciais que o contemplem assim como à sua família - para que possam adequar sua maneira de viver ao cotidiano social.

De modo análogo à preocupação da comunidade científica, a mídia (escrita e/ou falada) tem se interessado por esta problemática. Como exemplo, pode-se citar uma investigação que objetivou analisar as informações veiculadas nos principais meios de comunicação escrita do Brasil, ao longo de 1998, sobre a saúde relacionada ao uso de drogas ${ }^{3}$. Dentre os achados, identificou-se que os artigos acerca de derivados da coca (cocaína, crack e merla) foram alvo de um grande número de publicações que enfatizaram os prejuízos do uso e o aumento do consumo na população.

Ainda, notícias veiculadas por um jornal de grande circulação na região Sul do país, do dia 3 de agosto de 2009, destacaram que o crack se encontra entre os principais problemas de saúde pública do país e que está entre as epidemias mais assustadoras do Estado do Rio Grande do Sul, com cerca de 50 mil usuários e, aproximadamente, seis mortes diárias. Em virtude disso, deflagrou-se a campanha intitulada "Crack, nem pensar", tendo em vista que a dependência desta droga requer, além de um "amplo leque de ações focalizadas na prevenção e educação, um tratamento eficaz e resolutivo dos casos já existentes"4.

Assim, a partir deste cenário de proporções desafiantes, pode-se considerar que nas últimas três décadas a comunidade acadêmica tem procurado desenvolver estudos que possam clarear aspectos ainda pouco conhecidos acerca deste fenômeno, bem como definir diretrizes de atuação (desde a prevenção até o tratamento/reabilitação) e (re)orientar as políticas públicas na área da dependência química. Neste sentido, uma das formas de se conhecer as respostas científicas a determinado evento é revisando o que já foi produzido e publicado a respeito daquilo que se quer investigar. Este recurso tem sido utilizado, tanto a nível internacional ${ }^{5,6}$ quanto nacional ${ }^{3,7,8}, \mathrm{com}$ os mais variados objetivos, tais como esclarecer mitos a respeito do uso do crack, alertar os governos sobre a possibilidade de uma epidemia desta droga e/ou traçar o perfil dos usuários de crack.

Com base nas considerações decorrentes do aumento crescente do uso de crack na sociedade brasileira, o presente trabalho tem como questão de pesquisa: quais as contribuições do conhecimento produzido sobre o crack pelos cursos de mestrado e doutorado do país?

Isso porque entre as vulnerabilidades geradas pelo uso do crack que, não raramente, culminam com a dependência psíquica, estão os danos físicos pulmonares, o HIV/AIDS, as hepatites, a mortalidade, entre outros, o que justificam, por si só, a importância e a emergência de intervenções, bem como a ampliação de conhecimentos nesta área. Além dos aspectos já mencionados, destacam-se, também, o isolamento social, a marginalização/atos ilícitos, a violência, a degradação física e de caráter, o rompimento de laços afetivos com a família, o caos e o pânico 
coletivos que corroboram para minimizar a qualidade de vida, a perda de esperança na vida e as dificuldades de acesso aos serviços de saúde.

Tendo em vista as considerações anteriormente tecidas, este artigo tem como objetivo analisar as contribuições do conhecimento produzido sobre o crack pelos cursos de mestrado e doutorado brasileiros.

\section{Metodologia}

Trata-se de um estudo do tipo revisão sistemática, com base na modalidade revisão integrativa, a qual é definida como um método de avaliação crítica que agrupa resultados de estudos voltados a um mesmo tema/objeto, com vistas a analisar e sintetizar esses dados para desenvolver uma explicação mais abrangente de um fenômeno em específico ${ }^{9}$. Ainda, a revisão integrativa permite localizar, avaliar e sintetizar os resultados e as evidências disponíveis com vistas à sua incorporação na prática e desenvolve-se em cinco etapas ${ }^{9}$ : formulação do problema, coleta dos dados, avaliação dos dados coletados, análise e interpretação dos dados e apresentação dos resultados.

$\mathrm{Na}$ etapa de coleta, definiu-se o banco de dados, as estratégias de busca dos estudos e os critérios de inclusão e exclusão. Elegeu-se, então, o Banco de Teses da Coordenação Nacional de Aperfeiçoamento de Pessoal de Ensino Superior (CAPES) para a coleta dos dados, por se entender que ele agrupa as dissertações e as teses defendidas nos diversos Programas de Pós-Graduação brasileiros, concentrando 455.873 estudos entre os anos de 1987 e 2008. Como estratégia de busca utilizou-se a palavra "crack" no campo assunto do Banco de Teses da CAPES para se ter acesso à relação dos estudos, localizando-se um total de 100 dissertações e teses. Estabeleceuse como critérios de inclusão a necessidade destes trabalhos tratarem especificamente da temática do crack e de serem de qualquer área do conhecimento e excluiu-se aqueles que apenas citaram o crack em seus resumos.

Estas 100 produções passaram por uma leitura cuidadosa e criteriosa de seus resumos quando, então, 66 dissertações/teses foram excluídas por citarem o crack juntamente com outras drogas, não o tendo como foco das investigações e um trabalho foi excluído por não ter disponibilizado o resumo. Salienta-se que estes trabalhos não foram avaliados quanto ao mérito acadêmico e/ou metodológico (até porque não se configura proposta da revisão integrativa e nem obje- tivo deste artigo), tendo sido considerados como conhecimento de excelência por ter sua gênese em cursos stricto sensu reconhecidos pelo Ministério da Educação (MEC). Assim, 33 estudos foram incluídos e compuseram o material para esta revisão.

$\mathrm{Na}$ terceira etapa foi criado um instrumento para a avaliação dos trabalhos, bem como foram selecionadas as variáveis que permitiram o alcance do objetivo proposto. $\mathrm{O}$ instrumento foi elaborado na forma de quadros analíticos que agruparam dados de identificação do autor, título da dissertação/tese, ano de defesa, Programa de Pós-Graduação, Instituição de Ensino Superior (IES), região geográfica, objetivo e resultados das produções. Na quarta etapa, que se caracteriza pela síntese, comparação e discussão das variáveis extraídas das produções, foram construídas Tabelas (contendo números absolutos e relativos) e os resultados foram apresentados de forma descritiva. A quinta etapa, que consiste na apresentação dos resultados (seja em forma descritiva, de Tabelas e/ou gráficos), está contemplada na configuração deste artigo.

\section{Resultados}

O corpus de investigação foi composto por 33 trabalhos que investigaram o uso/abuso do crack, sendo 18 dissertações e 15 teses, distribuídas na Tabela 1 de acordo com o ano em que foram defendidas.

Em relação aos Programas de Pós-Graduação nos quais as dissertações/teses foram desenvolvi-

Tabela 1. Distribuição do total de dissertações e teses acerca do crack de acordo com o ano de defesa. Santa Maria, Brasil, 2010.

\begin{tabular}{cccc}
\hline Ano & Dissertações & Teses & $\mathbf{n}$ \\
\hline 1996 & - & 01 & 01 \\
1997 & 03 & 01 & 04 \\
1999 & - & 02 & 02 \\
2000 & 02 & 02 & 04 \\
2001 & 02 & 02 & 04 \\
2002 & 01 & - & 01 \\
2003 & 01 & - & 01 \\
2004 & 02 & 02 & 04 \\
2005 & 02 & 01 & 03 \\
2006 & 02 & 01 & 03 \\
2007 & 02 & 03 & 05 \\
2008 & 01 & - & 01 \\
Total & 18 & 15 & 33 \\
\hline
\end{tabular}


das, optou-se por agrupá-los levando em consideração as áreas de conhecimento ${ }^{10}$ a que estão vinculadas. A maioria dos estudos $(51,5 \%)$ pertencem à área das Ciências da Saúde em seus diferentes cursos stricto sensu, conforme Tabela 2.

Quando a análise recai sobre as IES que mais contribuíram com a produção do conhecimento relacionado ao crack, encontram-se como destaques a Universidade Federal de São Paulo e a Universidade de São Paulo, que foram responsáveis por $51,5 \%$ do total, conforme pode ser vislumbrado na Tabela 3.

Ao se estratificar os dados por região geográfica, constata-se que o sudeste brasileiro produziu 27 estudos ( 14 dissertações e 13 teses), respondendo por $81,8 \%$ do total. Em seguida, vieram as regiões nordeste na qual foram defendidos 3 trabalhos ( 1 de mestrado e 2 de doutorado) e sul (3 dissertações), o que representa 9,1\% cada. Analisando-se as temáticas mais abordadas e discutidas acerca da dependência pelo crack, observou-se que alterações orgânicas, tráfico de drogas e consumo de crack, HIV/AIDS, e modelos e estratégias de tratamento despontaram dentre as principais, e estão contempladas na Tabela 4.

\section{Discussão}

Esta revisão integrativa identificou 33 dissertações/teses que contemplaram a temática do crack e seus desdobramentos individuais, familiares e sociais, sendo 18 trabalhos de mestrado e 15 de doutorado. Considerando que o Banco de Teses da CAPES abriga estudos desde 1987 e que os primeiros relatos do uso desta droga entre a população brasileira data do início de 1990, constatou-se que as respostas advindas do conhecimento produzido pelos cursos stricto sensu não tardaram a aparecer. Isso porque, em 1996, é defendido o primeiro trabalho ${ }^{11}$ que, por meio de um estudo etnográfico, investigou o consumo de cocaína entre os baqueros (usuários de via endovenosa) e os craqueros (usuários de via pulmonar).

Ainda analisando o período de defesa dos estudos sobre o crack, vale ressaltar que a partir de

Tabela 2. Distribuição do total de dissertações e teses acerca do crack de acordo com a área do conhecimento e Programa de Pós-Graduação. Santa Maria, Brasil, 2010.

\begin{tabular}{|c|c|c|c|c|c|}
\hline $\begin{array}{c}\text { Área do } \\
\text { Conhecimento }\end{array}$ & Programa de Pós-Graduação & Dissertações & Teses & $\mathbf{n}$ & $\begin{array}{l}\text { Total da } \\
\text { Área }(\%)\end{array}$ \\
\hline \multirow[t]{10}{*}{ Ciências da Saúde } & Psiquiatria (e Psicologia Médica) & 02 & 03 & 05 & \multirow[t]{10}{*}{$17(51,5)$} \\
\hline & Anatomia Patológica e Patologia Clínica & - & 02 & 02 & \\
\hline & Doenças Infecciosas e Parasitárias & - & 02 & 02 & \\
\hline & Saúde Pública & 02 & - & 02 & \\
\hline & $\begin{array}{l}\text { Fonoaudiologia (Distúrbios da } \\
\text { Comunicação Humana) }\end{array}$ & - & 01 & 01 & \\
\hline & Medicina & - & 01 & 01 & \\
\hline & Neurologia & - & 01 & 01 & \\
\hline & Pediatria & - & 01 & 01 & \\
\hline & Ciências (Fisiopatologia Experimental) & 01 & - & 01 & \\
\hline & $\begin{array}{l}\text { Hebiatria: determinantes de saúde na } \\
\text { adolescência }\end{array}$ & 01 & - & 01 & \\
\hline \multirow[t]{7}{*}{ Ciências Humanas } & Psicologia & 06 & - & 06 & \multirow[t]{7}{*}{$15(45,5)$} \\
\hline & Psicologia (Psicologia Social) & 03 & - & 03 & \\
\hline & Psicobiologia & - & 02 & 02 & \\
\hline & Ciências Sociais & - & 01 & 01 & \\
\hline & $\begin{array}{l}\text { Psicologia Escolar e do Desenvolvimento } \\
\text { Humano }\end{array}$ & - & 01 & 01 & \\
\hline & Educação & 01 & - & 01 & \\
\hline & Psicologia Escolar & 01 & - & 01 & \\
\hline \multirow{2}{*}{$\begin{array}{l}\text { Ciências Sociais } \\
\text { e Aplicadas }\end{array}$} & Economia & 01 & - & 01 & $01(3,0)$ \\
\hline & Total & 18 & 15 & 33 & $33(100)$ \\
\hline
\end{tabular}


Tabela 3. Distribuição do total de dissertações e teses acerca do crack de acordo com a IES ao qual o curso stricto sensu está vinculado. Santa Maria, Brasil, 2010.

\begin{tabular}{|c|c|c|c|}
\hline IES & Dissertações & Teses & $\mathbf{n}$ \\
\hline $\begin{array}{l}\text { Universidade Federal de } \\
\text { São Paulo }\end{array}$ & 01 & 08 & 09 \\
\hline Universidade de São Paulo & 04 & 04 & 08 \\
\hline $\begin{array}{l}\text { Pontifícia Universidade } \\
\text { Católica de Campinas }\end{array}$ & 03 & - & 03 \\
\hline $\begin{array}{l}\text { Pontifícia Universidade } \\
\text { Católica de São Paulo }\end{array}$ & 03 & - & 03 \\
\hline $\begin{array}{l}\text { Universidade Federal da } \\
\text { Bahia }\end{array}$ & - & 02 & 02 \\
\hline $\begin{array}{l}\text { Universidade Estadual de } \\
\text { Campinas }\end{array}$ & - & 01 & 01 \\
\hline Fundação Oswaldo Cruz & 01 & - & 01 \\
\hline $\begin{array}{l}\text { Fundação Universidade de } \\
\text { Pernambuco }\end{array}$ & 01 & - & 01 \\
\hline $\begin{array}{l}\text { Pontifícia Universidade } \\
\text { Católica do Rio Grande do } \\
\text { Sul }\end{array}$ & 01 & - & 01 \\
\hline $\begin{array}{l}\text { Universidade de São Paulo } \\
\text { - Ribeirão Preto }\end{array}$ & 01 & - & 01 \\
\hline $\begin{array}{l}\text { Universidade do Vale do } \\
\text { Rio dos Sinos }\end{array}$ & 01 & - & 01 \\
\hline $\begin{array}{l}\text { Universidade Estadual } \\
\text { Paulista Júlio de Mesquita } \\
\text { Filho }\end{array}$ & 01 & - & 01 \\
\hline $\begin{array}{l}\text { Universidade Federal do } \\
\text { Rio Grande do Sul }\end{array}$ & 01 & - & 01 \\
\hline Total & 18 & 15 & 33 \\
\hline
\end{tabular}

Tabela 4. Distribuição das dissertações e teses acerca do crack de acordo com as temáticas investigadas. Santa Maria, Brasil, 2010.

\begin{tabular}{lccc}
\hline \multicolumn{1}{c}{ Temáticas } & Dissertações & Teses & n \\
\hline $\begin{array}{l}\text { Alterações orgânicas } \\
\text { Tráfico de drogas e consumo }\end{array}$ & 02 & 05 & 07 \\
de crack & 03 & 06 \\
$\begin{array}{l}\text { HIV/aids } \\
\text { Modelos e estratégias de }\end{array}$ & 04 & 04 & 05 \\
tratamento & & & 05 \\
$\begin{array}{l}\text { Prostituição, violência e } \\
\text { contravenção }\end{array}$ & 03 & - & 03 \\
$\begin{array}{l}\text { Mortalidade de usuários de } \\
\text { crack }\end{array}$ & 01 & 01 & 02 \\
$\begin{array}{l}\text { Reincidência no uso de } \\
\text { crack }\end{array}$ & 02 & - & 02 \\
$\begin{array}{l}\text { Tuberculose e uso de crack } \\
\text { Família de usuários de crack }\end{array}$ & 01 & 01 & 01 \\
$\begin{array}{l}\text { Motivações para o uso de } \\
\text { crack }\end{array}$ & 01 & - & 01 \\
Total & 18 & 15 & 33 \\
\hline
\end{tabular}

1999 sempre foram defendidos trabalhos sobre o tema e que o ano de 2007 se destacou por concentrar o maior número de estudos publicados, com duas dissertações e três teses. Este dado pode ser explicado pelo fato de, especialmente a partir da segunda metade da década de 1990, a mídia ter começado a explorar e a divulgar notícias relacionadas ao uso/abuso desta droga, o que alcançou o auge ao longo dos anos 2000 e repercutiu juntamente à comunidade científica. Ao aglutinar a produção encontrada, os dados revelam haver uma estabilização no conhecimento produzido, uma vez que entre 1996 e 2000 foram defendidas 11 dissertações/teses, entre 2001 e 2004 foram publicados 10 trabalhos e entre 2005 e 2008 foram produzidos 12 estudos sobre a temática, revelando uma preocupação, ainda que pequena, permanente, relacionada à epidemia do crack.

Quando estão em análise as áreas de conhecimento e os Programas de Pós-Graduação (PPG) que mais se envolveram na produção de saberes voltados às questões que se entrelaçam e constroem o uso do crack como um problema social, ressalta-se a área de Ciências da Saúde, responsável por 51,5\% das dissertações/teses e o PPG de Psiquiatria (e Psicologia Médica) com cinco estudos (dois de mestrado e três de doutorado) do total defendido na área. Este dado, embora importante, é também natural, uma vez que o uso/ abuso de substâncias dependógenas configura, historicamente, interesse do campo da saúde, especialmente do ramo da psiquiatria, a qual se caracteriza por ser uma disciplina específica da medicina responsável pela prevenção, atendimento, diagnóstico, tratamento e reabilitação das doenças mentais de origem patológica e/ou toxicológica; em virtude disso, o PPG de Psiquiatria se sobressaiu na produção deste conhecimento.

Há que se ressaltar a diversidade de cursos stricto sensu que se preocuparam em definir o crack como objeto das investigações científicas, pois em 10 PPG da área de conhecimento Ciências da Saúde foram defendidos trabalhos. Destaca-se, também, a área das Ciências Humanas que foi responsável pela construção de 15 estudos, representando $45,5 \%$ do total, dos quais o curso de Psicologia sobressaiu-se por ter produzido seis dissertações, dado que reflete o foco de interesse dessa ciência que são os comportamentos e os processos mentais dos seres humanos.

Em relação às 13 diferentes IES, quatro são Instituições Federais, as quais produziram 13 dissertações/teses, cinco são Instituições Estaduais responsáveis por 12 estudos concluídos e quatro são Instituições Particulares que construíram 
oito dissertações/teses. Dentre elas, destacam-se a Universidade Federal de São Paulo (UNIFESP) com a elaboração de um trabalho de mestrado e oito de doutorado e a Universidade de São Paulo (USP) com a produção de quatro dissertações e quatro teses, o que faz com que o Estado de São Paulo se sobressaia na construção stricto sensu voltada a esta temática, com 26 trabalhos $(78,8 \%)$. Em consonância com estes achados, a região Sudeste é a detentora da maior produção de dissertações/teses $(81,8 \%)$, seguida do Nordeste e Sul brasileiros (18,2\% cada).

O fato de as IES de São Paulo serem as responsáveis pela maior parte do conhecimento produzido acerca do uso/abuso do crack pode ser explicado por terem ocorrido nesta região as apreensões iniciais desta droga, assim como por ser uma problemática disseminada há, aproximadamente, duas décadas neste Estado (sendo mais recente em outros), repercutindo em transtornos para as famílias (de usuários e de não usuários), para as comunidades (tanto de regiões nobres como de favelas) e para as instituições sociais (das áreas da saúde, segurança, educação e outras). Quanto à supremacia da região Sudeste, há que se considerar que ela abriga $50,15 \%{ }^{12}$ do total de cursos stricto sensu brasileiros, o que auxilia a justificar o número de estudos. Em contrapartida, chama atenção a ausência de dissertações/teses do centro-oeste e norte do país, regiões que precisam investir no desenvolvimento de pesquisas a fim de compreender as nuances e as particularidades que o fenômeno do crack assume entre suas populações.

Encontrou-se, ainda, 10 categorias temáticas diferentes representativas do interesse acadêmico voltado à epidemia do crack, as quais serão apresentadas abaixo:

\section{Alterações orgânicas}

Foi o tema mais presente entre as dissertações e teses, totalizando sete estudos ${ }^{13-19}$. Destes, os usuários de crack, de uma maneira geral, foram sujeitos de estudos do tipo caso-controle que visaram avaliar a função vestibular ${ }^{13}$, caracterizar o desempenho neuropsicológico ${ }^{14}$ e estudar as queixas auditivas e os achados do potencial evocado auditivo do tronco encefálico ${ }^{15}$. Os recém-nascidos (RN) e suas mães foram pesquisados com vistas a comparar a relação entre o baixo peso ao nascer e os níveis da droga presentes no mecônio, na urina e no cabelo de ambos ${ }^{16}$. Os adolescentes foram sujeitos de um estudo transversal-observacional que objetivou determinar a ocorrência de transtornos vocais e larín- geos dos usuários ${ }^{17}$. Outros dois estudos experimentais envolveram a avaliação dos efeitos da inalação crônica de crack na espermatogênese ${ }^{18} \mathrm{e}$ no aparelho respiratório ${ }^{19}$ de camundongos.

Um estudo ${ }^{13}$ encontrou que a maioria dos usuários de crack apresentou sintomas que indicam diagnóstico de síndrome vestibular periférica do tipo irritativo ou deficitário, devendo ser um exame a ser incluído na avaliação clínica destes indivíduos. Outra tese ${ }^{14}$ identificou que os usuários de crack apresentaram desempenho mais alto na ansiedade e mais baixo nos testes cognitivos, sugerindo que este padrão de uso (fumado) é mais deletério devido seu poder de ação no Sistema Nervoso Central (SNC). Já uma dissertação ${ }^{15}$ concluiu, ao utilizar o exame de audiometria de tronco encefálico, que não houve diferença estatisticamente significativa quanto à latência, amplitude, morfologia e intervalos interpicos entre os grupos de usuários de crack e os não usuários.

Outro estudo ${ }^{16}$ encontrou que o número de RN de baixo peso nascidos de mães usuárias de crack foi maior do que os de mães com outros fatores reconhecidamente predisponentes ao baixo peso (cardiopatia ou hipertensão), sendo que eles precisam ser identificados (por exemplo, através do mecônio por não ser invasivo e ser menos constrangedor) com vistas a um melhor acompanhamento e tratamento pós-natal. Uma dissertação ${ }^{17}$ identificou transtornos vocais do tipo rouquidão/aspereza e tensão (predominantemente de grau 1), bem como transtornos laríngeos atribuíveis, relacionados e agravados pelo uso do crack entre adolescentes.

Uma tese de doutorado ${ }^{18}$ concluiu que houve aumento de degeneração celular germinativa nos camundongos expostos à fumaça da cocaína/cra$\mathrm{ck}$, induzindo alterações na espermatogênese, em especial e com maior toxicidade nos animais mais jovens. Outro estudo com cobaias ${ }^{19}$ encontrou que a inalação de crack causa alterações no aparelho respiratório (do nariz até o parênquima pulmonar), demonstradas pela diminuição do epitélio, aumento de muco no epitélio nasal e brônquico, vasoconstrição das arteríolas pulmonares, aumento de hemossiderina pulmonar e de macrófagos e neutrófilos pulmonares.

\section{Tráfico de drogas e consumo de crack}

Representa outra temática que tem despertado o interesse de estudiosos e pesquisadores, demonstrado pela construção de seis dissertações/ teses $^{11,20-24}$, todas tendo como cenário o Estado de São Paulo. Dois estudos recorreram à aborda- 
gem etnográfica a fim de analisar como ocorre o consumo de drogas entre os usuários de via endovenosa e aqueles de via pulmonar ${ }^{11}$, bem como caracterizar a cultura do crack de forma a identificar o perfil do usuário e o seu padrão de uso ${ }^{20}$. A fenomenologia social de Alfred Schütz foi outra abordagem adotada com o intuito de compreender a condição dos trabalhadores rurais que se dedicam ao corte da cana-de-açúcar e os aspectos histórico-culturais relacionados ao uso/abuso de $\operatorname{crack}^{21}$. Ainda de perspectiva qualitativa, outros dois estudos envolveram adolescentes e jovens com vistas a investigar as relações sociais daqueles que vivem em situação de rua e consomem crack $^{22}$ e traçar o percurso de vida daqueles que "trabalham" vendendo drogas ilícitas ${ }^{23}$. De cunho quantitativo, tem-se o estudo ${ }^{24}$ que utilizou dados fornecidos pela Secretaria de Segurança Pública do Estado de São Paulo sobre as quantidades de drogas apreendidas ao longo de 2001.

Um estudo ${ }^{20}$ encontrou que a cultura de crack tem sofrido profundas modificações, o que pode justificar a não-adesão dos usuários às medidas de intervenção disponíveis; que o uso, embora ainda seja, em sua maioria, compulsivo e submetido a uma série de riscos, apresenta o padrão controlado e que a cultura parece ser mais um produto do crack em si do que do contexto social em que está inserido. Já uma dissertação ${ }^{21}$ concluiu que os trabalhadores do corte da cana e usuários de crack relataram ruptura com os vínculos familiares e com a religião, condições precárias de sobrevivência e dúvidas sobre a droga em sua relação com o trabalho e as questões sociais, apontando para a necessidade de se conhecer os porquês do consumo desta substância.

A tese $\mathrm{e}^{23}$ encontrou uma complexidade existente nos discursos relacionados ao mundo do trabalho (inserção dos jovens no comércio de drogas ilícitas), ao contrato social (as formas que regulam as relações sociais existentes no tráfico) e à violência (tanto da polícia, quanto dos traficantes). Já outros estudos ${ }^{11,22,24}$ não apresentaram, em seus resumos, os resultados e/ou conclusões das investigações que permitissem descrevê-los.

\section{HIV/Aids}

Esta temática, em sua correlação com o crack, também foi elaborada para contribuir com o conhecimento construído na área ${ }^{25-29}$. Os perfis e os comportamentos de risco de usuários de crack e as possibilidades/estimativas de infecção pelo HIV foram foco de três teses ${ }^{25-27}$. As mulheres usuárias de crack foram sujeitos das outras duas produções, seja para identificar suas característi- cas clínico-epidemiológicas e os aspectos bioéticos envolvidos com o $\mathrm{HIV}^{28}$ ou, então, para avaliar as relações entre as profissionais do sexo e os caminhoneiros, bem como o papel destes na dinâmica da epidemia da $\operatorname{AIDS}^{29}$.

Uma tese ${ }^{25}$ encontrou que $90 \%$ dos usuários de crack investigados eram do sexo masculino, com idade média de 27 anos, sendo que $62 \%$ eram solteiros, $62 \%$ só estudaram até o primeiro grau (completo ou incompleto), 30\% estavam trabalhando, 46\% estavam desempregados, 54\% moravam na casa dos pais, $34 \%$ ganhavam menos que um salário mínimo por mês, mas 21\% ganhavam mais que 5 salários mínimos. Ainda, os usuários consumiam 9,5 pedras de crack por dia, $63 \%$ usavam a pedra todos os dias, $56 \%$ tinham sido presos, $13 \%$ tinham trocado sexo por dinheiro ou droga e os pacientes com HIV/AIDS fizeram sexo com um número menor de pessoas, porém não usaram preservativos com os parceiros casuais com maior frequência do que os indivíduos HIV negativos.

Um estudo ${ }^{26}$ encontrou predomínio de homens $(95,7 \%)$ entre os usuários de crack pesquisados, brancos $(70,8 \%)$, jovens (mediana de 26 anos), com antecedentes de detenção (54\%) e moradia na rua $(24,9 \%)$. Concluiu que eles não apresentavam risco maior de se infectarem pelo HIV, HCV ou Treponema pallidum, mas reforça a necessidade de investimentos e de desenvolvimento de estratégias de prevenção. Outra tese ${ }^{27}$ caracterizou os usuários como sendo predominantemente do sexo masculino (98\%), com menos de 30 anos $(73 \%)$, com vínculo empregatício (51\%), policonsumistas de drogas (76\%), com atividade sexual semanal (47\%), fazendo pouco uso de preservativos (mesmo no caso de prostituição), com informações sobre AIDS (a quase totalidade) e que $51 \%$ não modificaram seu comportamento desde o surgimento desta. Indica a necessidade de se criarem estratégias preventivas ao HIV/AIDS para os usuários de cocaína (em suas diversas formas) e que o consumo de crack está se disseminando entre os usuários de drogas injetáveis.

Já uma tese ${ }^{28}$ concluiu que a média de idade das mulheres investigadas com HIV/AIDS e usuárias de crack era de 22 anos, sendo 91,2\% afrodescendentes, 56\% desempregadas, com início de vida sexual aos 14 anos (66,9\%), com 10,9 parceiros em média, sendo que $58 \%$ não referiram o uso de preservativo nos últimos 30 dias e que $36,8 \%$ afirmaram trocar sexo por dinheiro ou drogas. Outro estudo ${ }^{29}$, envolvendo mulheres profissionais do sexo e usuárias de crack, encontrou que elas apresentam baixa autopercepção 
de risco ao HIV e relataram experiências de violência física e sexual (especialmente relacionadas à negociação do uso do preservativo). Além disso, constatou que muitas delas são moradoras de rua ou favelas e dificilmente acessam os serviços de saúde (centros de testagem e aconselhamento, pré-natal, maternidades e outros), recomendando intervenções multifacetadas e culturalmente apropriadas para a prevenção da infecção pelo HIV nesta população.

\section{Modelos e estratégias de tratamento}

Temática contemplada em cinco estudos e que demonstrou os diferentes recursos que vêm sendo empregados para melhorar e/ou (re)adequar as formas de tratamento adotadas. Estudo da área da psicologia apresentou três métodos (os 12 passos dos narcóticos anônimos, os 12 passos para os cristãos e a prevenção da recaída) mais empregados por profissionais que atuam em consultórios ou clínicas especializadas no atendimento a usuários de $\operatorname{crack}^{30}$. Outros modelos e estratégias encontrados foram: a utilização de jogo terapêutico para jovens usuários da droga ${ }^{31}$; a utilização do modelo transteórico de mudança de comportamento em tratamentos para dependência química na versão adaptada para a língua brasileira $^{32}$; a estratégia de redução de danos $^{33}$; e, a proposta de escuta psicanalítica no tratamento de internos de uma comunidade terapêutica ${ }^{34}$.

Uma dissertação ${ }^{30}$ encontrou que os diferentes modelos de tratamento para os usuários de crack devem levar em consideração três núcleos básicos, quais sejam: a dependência química (como doença e suas consequências), os pressupostos usados na escolha do modelo de tratamento e a relação existente entre os modelos escolhidos e as estratégias usadas pelos profissionais no atendimento. Já um estudo ${ }^{31}$ constatou que o jogo terapêutico (denominado "Jogo da Escolha") aumentou a motivação dos usuários de crack que estão ambivalentes em relação a parar de usar a droga, aumentou o senso de autoeficácia e, efetivamente, diminuiu o consumo de crack, podendo sua aplicação ser indicada no início do tratamento (como motivador) ou como um pré-tratamento para os usuários que estão em uma fila de espera para atendimento.

Outro estudo ${ }^{32}$ constatou validade de alta intensidade no uso total (24 itens) da University of Rhode Island Change Assesment (URICA) adaptação brasileira -, bem como para suas subescalas, podendo ser usada em estudos que se proponham a avaliar a motivação para mudanças no hábito de usar drogas ilícitas. A tese ${ }^{33}$ si- naliza para a dificuldade de implementar estratégias de redução de danos aos usuários de crack (o que passa pela forma de financiamento e de execução dos projetos, e até por questões de ordem moral) e propõe uma discussão ampla com a sociedade sobre esta estratégia, com vistas a garantir os direitos à saúde dos usuários de drogas. Por fim, uma dissertação $0^{34}$ encontrou que a escuta (de acordo com a psicanálise) revela-se como importante e efetiva ferramenta no processo de mudança da posição subjetiva dos usuários de crack, permeando desde a adesão ao programa de tratamento até a possibilidade de fazêlo olhar para si e perceber mudanças (internas e externas) na disposição de deixar a droga.

\section{Prostituição, violência e contravenção}

Esta temática esteve presente em três dissertações que trataram, em linhas gerais, de compreender o processo de construção do usuário de crack, suas narrativas do vício e as relações com a violência ${ }^{35}$, de analisar o momento limítrofe entre o estar na lei e o estar fora da lei vivenciado por crianças e adolescentes usuários de cra$\mathrm{ck}^{36} \mathrm{e}$ de conhecer as relações que se estabelecem entre a prática da prostituição feminina e o uso de crack $^{37}$. A dissertação ${ }^{37}$ concluiu que o consumo de crack representa uma forma de viver no cotidiano da rua e que existem algumas formas (mesmo que incipientes) de cuidados com a saúde e o corpo dentro deste estilo de vida, recomendando a implantação de estratégias de redução de danos para o uso da droga. Os outros estudos $^{35,36}$ não continham resultados/conclusões em seus resumos que permitissem descrevê-los.

\section{Mortalidade de usuários de crack}

Foi tema de dois estudos de coorte que acompanhou os usuários de crack ao longo de cinco anos com o intuito de avaliar a mortalidade relacionada ao consumo da droga ${ }^{38}$, assim como a evolução dos padrões de consumo, sociodemográficos e de mortalidade relacionados ao crack $^{39}$. O primeiro ${ }^{38}$ identificou que, após cinco anos de acompanhamento, houve melhora da abstinência por motivos aparentemente não relacionados à procura por tratamento entre os usuários de crack, que a mortalidade (principalmente por homicídio e pela AIDS) é maior do que na população em geral e que o consumo de drogas endovenosas e a presença de desemprego na internação estão diretamente relacionados ao aumento da mortalidade. Já uma tese ${ }^{39}$ apontou que, após o mesmo período de acompanhamento, houve uma melhora nos níveis de emprego entre os usu- 
ários de crack e que a chance de sobrevida foi de $80 \%$, com uma taxa de mortalidade bruta anual de 35,11 óbitos/1.000 habitantes.

\section{Reincidência no uso de crack}

Esta temática foi foco de duas dissertações que se desenvolveram no PPG de Psicologia e tiveram por objetivo: identificar as variáveis críticas da reincidência do uso de crack e o tempo médio de recaída ${ }^{40}$ e analisar as contingências do comportamento de recaída de usuários de crack que estiveram em uma comunidade terapêuti$\mathrm{Ca}^{41}$. Outro estudo ${ }^{40}$ constatou que a falta de habilidade de enfrentamento de situação de autorrisco constituiu-se na variável crítica da reincidência e que o período crítico para a recaída foram os primeiros seis meses (em especial os dois primeiros), recomendando a necessidade de formação de uma rede de apoio ao usuário e sua família durante e após o tratamento, envolvendo, além de uma equipe especializada, colegas, empregadores e a sociedade como um todo. A outra dissertação ${ }^{41}$ concluiu que o início do uso da droga ocorre na infância e na adolescência, que os fatores familiares e o convívio em ambientes com usuários de drogas são variáveis da história de vida e que é necessário, além de modificações de comportamento no sentido de aumentar o autocontrole, o estabelecimento de limites e de resistência à frustração.

\section{Tuberculose e uso de crack}

Foi tema de uma tese que avaliou o padrão de uso da droga entre usuários que se encontram hospitalizados em serviços de saúde da grande São Paulo e a dimensão da presença de tuberculose nestes ${ }^{42}$. Concluiu que não houve prevalência aumentada de tuberculose infecção ou doença nos usuários de crack e que seu rastreamento ainda necessita de método efetivo, eficiente e sensível.

\section{Família de usuários de crack}

Esta temática despertou interesse de estudiosos da área da psicologia com vistas a averiguar a relação entre stress e qualidade de vida de progenitores de usuários da droga em momentos anteriores e posteriores a uma intervenção do tipo grupo de apoio ${ }^{43}$. O estudo encontrou que as mulheres progenitoras têm stress significativamente maior do que os homens, que quanto maior o stress dos progenitores menor a qualidade de vida social, que as maiores dificuldades que tiveram desde que souberam do uso de drogas do/a filho/a foi a agressividade, o roubo e o furto por parte dos/as mesmos/as. Ainda, que os pensamentos mais frequentemente mencionados foram o medo de morte e de prisão do/a filho/a, e que a qualidade de vida da saúde teve $100 \%$ de prejuízo.

\section{Motivações para o uso de crack}

Foi foco de uma dissertação vinculada ao PPG da Educação, tendo como sujeitos os adolescentes dependentes da droga e visou investigar as possíveis motivações para o consumo do cra$\mathrm{ck}^{44}$. Este estudo concluiu que mais do que as perdas ocasionadas pelo uso disfuncional da droga, a conscientização da dependência pareceu relacionar-se com a incoerência entre a autoidentificação e os papéis de poder, a identidade negada de dependente e a manutenção de papéis infantis e ambivalentes.

Constatou-se que os saberes stricto sensu produzidos pelos pesquisadores brasileiros são ainda incipientes, pontuais e pouco resolutivos, embora promissores frente às demandas e às implicações que a epidemia do crack impõe à sociedade. Ainda, este conhecimento encontrase circunscrito a determinadas regiões geográficas, mesmo sendo um problema nacional. Ressalta-se, desta forma, a necessidade de descentralização do conhecimento, tendo em vista que o Brasil apresenta regiões muito diferenciadas em termos populacionais, estruturais e organizacionais, estando as respostas à epidemia do crack relacionadas às particularidades, às potencialidades e às limitações de cada unidade federativa. E isso poderá ser alcançado com maiores (e melhores) investimentos por parte dos órgãos de fomento às pesquisas (estaduais e nacionais), bem como com a qualificação dos pesquisadores e com a implantação de cursos de mestrado e doutorado em regiões ainda pouco desenvolvidas (caso do centro-oeste e do norte brasileiros).

\section{Conclusões}

Esta investigação revelou que, embora o consumo de crack constitua-se um fenômeno disseminado na sociedade e, constantemente, noticiado nos veículos de comunicação em virtude dos transtornos e desafios que tem imposto às famílias, comunidades e governos, a produção científica dos cursos de mestrado e doutorado do país não tem acompanhado a preocupação generalizada. Isso porque os 33 estudos que investigaram o uso desta droga, em um universo de 455.873 trabalhos que compõem o Banco de Te- 
ses da CAPES, representam, apenas, 0,007\% do total, indicando a necessidade de maiores investimentos e pesquisas na área.

Identificou-se que há um equilíbrio na elaboração de dissertações e teses, que a partir de 1999 sempre foram defendidos trabalhos acerca da epidemia do crack e que o ano de 2007 destacou-se como o detentor de maior acúmulo da produção. Ainda, que a área do conhecimento das Ciências da Saúde e, mais especificamente, o Programa de Pós-Graduação em Psiquiatria (e Psicologia Médica) foram os responsáveis pelo maior número de estudos voltados ao consumo desta droga.

A Universidade Federal de São Paulo e a Universidade de São Paulo foram as IES que mais contribuíram para a construção de dissertações/ teses $(51,5 \%)$, aglutinando a produção acerca da epidemia do crack na região Sudeste brasileira $(81,8 \%)$, com especial destaque para o Estado de São Paulo $(78,8 \%)$, locus a partir do qual esta droga propagou-se para as outras regiões do país. As temáticas mais investigadas estiveram relacionadas a alterações orgânicas, tráfico de drogas e consumo de crack, HIV/AIDS, e modelos e estratégias de tratamento, responsáveis por $69,7 \%$ do total analisado.

Os objetos que têm sido investigados dizem respeito, em sua grande maioria, aos campos da assistência, tratamento e recuperação, sendo fundamental que avancem para os da promoção da saúde e prevenção do agravo, a fim de que se possa ter diferentes estratégias intervencionistas frente ao consumo da droga. As temáticas, na quase totalidade, referem-se a problemas orgâ- nicos e a doenças, frutos de um paradigma ainda em voga que, guardadas suas potencialidades, tem se mostrado insuficiente a responder, com eficiência, a fenômenos que envolvem aspectos históricos, sociais, ambientais, econômicos e culturais, como é o caso da epidemia do crack. Além disso, percebe-se a necessidade de outras áreas do conhecimento (por exemplo, as Ciências Humanas e as Ciências Sociais e Aplicadas) assumirem as problemáticas que envolvem a temática do crack como prioritárias, com vistas a contribuírem com os resultados que já vêm sendo produzidos, especialmente pelas Ciências da Saúde.

Portanto, os resultados evidenciam que a temática do crack, apesar de assustadora, não se constitui numa área de interesse para as investigações e tão pouco para as intervenções, tanto em sua dimensão preventiva quanto curativa e reabilitadora. $\mathrm{O}$ estudo se mostra pertinente, nessa perspectiva, pela possibilidade de evidenciar o conhecimento já produzido, mas, sobretudo, pela possibilidade de trazer à tona as lacunas, bem como as incertezas e os temores associados à problemática. Além disso, alerta para a necessidade de se ampliar os horizontes da pesquisa e de buscar respostas para questões emergentes que assolam a sociedade. É preciso que pesquisadores, estudiosos e profissionais em geral, estejam engajados na busca de estratégias integradas e integradoras, centradas no ser humano que, momentaneamente ou não, vê-se impelido ao uso/abuso do crack, mas que deve ser vislumbrado pelos profissionais, gestores e serviços de saúde como um ser singular, familiar e multidimensional.

\section{Colaboradores}

D Schaurich R, DS Backes, HMB Freitas, C Zamberlan, MH Gelhen e JS Colomé participaram igualmente de todas as etapas de elaboração do artigo. 


\section{Referências}

1. Kessler F, Pechansky F. Uma visão psiquiátrica sobre o fenômeno do crack na atualidade. Rev Psiquiatr RS 2008; 30(2):96-98.

2. Centro Brasileiro de Informações sobre Drogras Psicotrópicas (CEBRID). Crack: últimos 20 anos - I Fórum Internacional. São Paulo, 26 e 27 de março de 2009. [acessado 2009 abr 17]. Disponível em: http://www.unifesp.br/eventos/forumcrack/.

3. Noto AR, Baptista MC, Faria ST, Nappo AS, Galduróz JCF, Carlini EA. Drogas e saúde na imprensa brasileira: uma análise de artigos publicados em jornais e revistas. Cad Saude Publica 2008; 19(1):69-79.

4. Etchichury C, Gava R. Um marco no flagelo do crack. Zero Hora 2009; 14 abr. p. 15.

5. Cornish JW, O’Brien. Crack cocaine abuse: an epidemic with many public health consequences. Annu Rev Public Health 1996; 17:259-273.

6. Haasen C, Krausz M. Myths versus evidence with respect to cocaine and crack: learning from the US experience. Eur Addict Res 2001; 7(4):159-160.

7. Duailibi LB, Ribeiro M, Laranjeira R. Profile of cocaine and crack users in Brazil. Cad Saude Publica 2008; 24(Supl.4):545-557.

8. Duailibi LB, Ribeiro M, Laranjeira R. Perfil dos usuários de cocaína e crack no Brasil. Associação Brasileira de Estudos do Álcool e outras Drogas [site na Internet]. [acessado $2009 \mathrm{dez}$ 18]. Disponível em: http://www.abead.com.br/artigos/arquivos/perfil_ usuario_coca_crack.pdf

9. Cooper HM. Integrative research: a guide for literature reviews. $2^{\text {a }}$ ed. London: SAGE publication; 1989.

10. Brasil. Ministério da Ciência e Tecnologia. Conselho Nacional de Desenvolvimento Científico e Tecnológico. Áreas do conhecimento. [site na Internet]. [acessado 2010 fev 5]. Disponível em: http://www. cnpq.br/areasconhecimento/index.htm

11. Nappo AS. Baquêros e craquêros: um estudo etnográfico do consumo de cocaína na cidade de São Paulo [tese]. São Paulo (SP): Universidade Federal de São Paulo; 1996.

12. Brasil. Ministério da Ciência e Tecnologia. Conselho Nacional de Desenvolvimento Científico e Tecnológico. Geocapes: dados estatísticos. [site na Internet]. [acessado 2010 mar 3]. Disponível em: http:/ /geocapes.capes.gov.br/geocapesds/

13. Taguchi CK. Da função vestibular em usuários de crack: pesquisa vecto-electronistagmografica [tese]. São Paulo (SP): Universidade Federal de São Paulo; 1997.

14. Nassif SLS. Avaliação neuropsicológica de usuários crônicos de cocaína não injetável, fora do período de intoxicação aguda [tese]. São Paulo (SP): Universidade Federal de São Paulo; 2001.

15. Nigri LF. Potenciais evocados auditivos do tronco encefálico em usuários de crack e múltiplas drogas [dissertação]. São Paulo (SP): Universidade de São Paulo; 2002.

16. Wong A. Cocaina/crack, canabinóides, anfetaminas e opiáceos no cabelo, urina e mecônio de recém-nascidos e baixo peso e a correlação destes no cabelo e urina das mães [tese]. São Paulo (SP): Universidade de São Paulo; 2001.
17. Balata PMM. Os transtornos vocais e laríngeos em adolescentes e jovens usuários de drogas fumadas [dissertação]. Recife (PE): Fundação Universidade de Pernambuco; 2007.

18. Herculiani PP. Efeitos da inalação crônica de cocaína "crack" no aparelho respiratório de camundongos [tese]. São Paulo (SP): Universidade de São Paulo; 2007.

19. Zorzetto JC. Avaliação dos efeitos da inalação crônica de cocaína crack na espermatogênese de camundongos [tese]. São Paulo (SP): Universidade de São Paulo; 2007.

20. Oliveira LG. Avaliação da cultura do uso de crack após uma década de introdução da droga na cidade de São Paulo [tese]. São Paulo (SP): Universidade Federal de São Paulo; 2007.

21. Andrade AF. Cana e crack: sintoma ou problema? Um estudo sobre os trabalhadores no corte de cana e o consumo de drogas/crack [dissertação]. São Paulo (SP): Pontifícia Universidade Católica de São Paulo; 2003.

22. Rigon JM. Adolescentes de classes populares e consumo de crack em Ribeirão Preto/SP [dissertação]. Ribeirão Preto (SP): Universidade de São Paulo/Ribeirão Preto; 2000.

23. Feffermann M. Vidas arriscadas: um estudo sobre os jovens inscritos no tráfico de drogas em são Paulo [tese]. São Paulo (SP): Universidade de São Paulo; 2004.

24. Castro FP. A economia das drogas na cidade de São Paulo [dissertação]. Araraquara (SP): Universidade Estadual Paulista Júlio de Mesquita Filho; 2007.

25. Dunn J. Usuários de cocaína: seus perfis, padrões de uso e comportamentos de risco para a transmissão do vírus HIV [tese]. São Paulo (SP): Universidade Federal de São Paulo; 1999.

26. Turchi MD. Perfil de risco e estimativa de ocorrência de infecções de transmissão sangüinea ou sexual HIV, Hepatite B, Hepatite C, HTLV I/II e Sífilis - entre usuários de cocaína, em São Paulo [tese]. São Paulo (SP): Universidade Federal de São Paulo; 2000.

27. Azevedo RCS. Usuários de cocaína e AIDS: um estudo sobre comportamento de risco [tese]. Campinas (SP): Universidade Estadual de Campinas; 2000.

28. Nunes CLX. Características clínico-epidemiológicas e aspectos bioéticos relacionados a infecção pelo HIV/ AIDS em mulheres na Bahia [tese]. Salvador (BA): Universidade Federal da Bahia; 2004.

29. Malta MS. Uso de drogas \& HIV/AIDS entre profissionais do sexo e caminhoneiros do sul do país: implicações para a saúde pública e possíveis intervenções [dissertação]. Rio de Janeiro (RJ): Fundação Oswaldo Cruz; 2005.

30. Silva AF. A dependência química e o atendimento oferecido: comparação de modelos de tratamento e seu respectivo significado para os profissionais de psicologia que atendem a dependentes químicos [dissertação]. São Paulo (SP): Pontifícia Universidade Católica de São Paulo; 2001.

31. Williams AV. Desenvolvimento e avaliação da eficácia de um jogo terapêutico para jovens usuários de drogas [dissertação]. Porto Alegre (RS): Universidade Federal do Rio Grande do Sul; 2006. 
32. Szupszynski KPDR. Adaptação brasileira da University of Rhode Island Change Assesment (URICA) para usuários de substâncias psicoativas ilícitas [dissertação]. Porto Alegre (RS): Pontifícia Universidade Católica do Rio Grande do Sul; 2006.

33. Domanico A. Craqueiros e cracados: bem vindo ao mundo dos nóias! Estudo sobre a implementação de estratégias de redução de danos para usuários de crack nos cinco projetos-piloto do Brasil [tese]. Salvador (BA): Universidade Federal da Bahia; 2006.

34. Saft L. A escuta de usuários de crack no contexto de comunidade terapêutica [dissertação]. São Leopoldo (RS): Universidade do Vale do Rio dos Sinos; 2008.

35. Lima LCA. "Vício" e a violência: o cotidiano do crack e as narrativas do vício [dissertação]. São Paulo (SP): Pontifícia Universidade Católica de São Paulo; 1997.

36. Feffermann M. Na fronteira da lei e do fora da lei: um estudo sobre o discurso de crianças e adolescentes na periferia do município de São Paulo [dissertação]. São Paulo (SP): Universidade de São Paulo; 1997.

37. Silva SL. Mulheres da luz: uma etnografia dos usos e preservação no uso do crack [dissertação]. São Paulo (SP): Universidade de São Paulo; 2000.

38. Araújo MR. Estudo de seguimento com usuários de crack: mortalidade durante cinco anos [dissertação]. São Paulo (SP): Universidade Federal de São Paulo; 2001.

39. Araújo MR. Estudo de seguimento com usuários de crack: mortalidade durante cinco anos [tese]. São Paulo (SP): Universidade Federal de São Paulo; 2005.
40. Oliveira LC. Variáveis críticas de reincidência as drogas em farmacodependentes [dissertação]. Campinas (SP): Pontifícia Universidade Católica de Campinas; 1997.

41. Dalbem E. Análise do comportamento de reincidência através de depoimentos de dependentes químicos [dissertação]. Campinas (SP): Pontifícia Universidade Católica de Campinas; 2004.

42. Ferreira Filho OF. Estimativa da prevalência de tuberculose infecção e doença entre usuários de cocaína, internados em alguns serviços hospitalares da grande São Paulo [tese]. São Paulo (SP): Universidade Federal de São Paulo; 1999.

43. Vicentin VF. Stress e qualidade de vida dos progenitores de usuários de cocaína e crack [dissertação]. Campinas (SP): Pontifícia Universidade Católica de Campinas; 2004.

44. Baptista GC. Adolescência e drogas: um estudo exploratório na região metropolitana de São Paulo [dissertação]. São Paulo (SP): Universidade de São Paulo; 2005.

Artigo apresentado em 23/03/2011

Aprovado em 06/04/2011

Versão final apresentada em 31/05/2011 